\title{
Investigación sobre la eficacia de la publicidad en videojuegos: estado de la cuestión
}

Research on the Effectiveness of Advertising in Videogames: State of the Art 
RESUMEN

\section{Clasificación JEL: \\ M37 \\ Key words:}

Publicidad,

videojuegos,

emplazamiento de

producto,

in-game advertising,

advergaming,

análisis de contenido

La publicidad a través de videojuegos está adquiriendo un creciente protagonismo como herramienta de las comunicaciones de marketing de las organizaciones. Ya sea mediante el uso del emplazamiento de producto en videojuegos, del in-game advertising o del advergaming, la utilización de los videojuegos como herramienta de las comunicaciones de marketing ha experimentado un importante incremento en los últimos años dentro de los presupuestos de marketing de las organizaciones. Junto a este incremento en la praxis comercial, en los últimos años también se ha producido un aumento de las investigaciones académicas que han centrado su atención en el uso de los videojuegos como herramienta publicitaria. El presente trabajo exploratorio analiza 19 artículos académicos que han estudiado diferentes áreas que exploran la eficacia publicitaria en el uso de los videojuegos como herramienta publicitaria. El objetivo principal de este trabajo es identificar las principales líneas de investigación así como las principales metodologías y factores empleados. Utilizando el análisis de contenido como herramienta de investigación, el artículo muestra un estado de la cuestión de la investigación académica sobre la eficacia de la publicidad a través de videojuegos y destaca potenciales áreas de interés en las que se ha detectado una escasez de investigación académica que, por tanto, ofrecen nuevas oportunidades a los investigadores.

\section{ABSTRACT}

\section{JEL Classification:}

M37

\section{Palabras clave:}

Advertising, video games, product placement, in-game advertising, advergaming, contents analysis
Advertising through videogames is acquiring increasing prominence as a marketing communications tool for organisations. Be it through the use of product placement in videogames, in-game advertising or advergaming, the use of videogames as a marketing communications tool has seen an important increase in the last few years in the marketing budgets of organisations. Together with this increase in the commercial implementation, there has also been an increase in academic research that has centered its attention on the use of video games as an advertising tool. The present exploratory work analyses 19 academic articles that have studied different areas of advertising efficacy in the use of video games as an advertising tool. The main objective of this work is to identify the principal lines of research as well as the principal methodologies and factors used. Using the contents analysis as an investigative tool, the article shows the state of the matter of the academic research on the efficacy of advertising through video games and highlights potential areas of interest where there is a scarcity of academic research and, therefore, offers new opportunities to researchers. 


\section{Introducción}

El importante cambio que está experimentando el panorama mediático en los últimos años como fruto de la irrupción de nuevas tecnologías en la difusión de contenidos, unido a los importantes cambios en el consumo de medios por parte de los consumidores (Díaz Nosty, 2006), está obligando a las organizaciones a replantear sus estrategias de comunicación como forma de adaptarse al mercado. En este sentido, en los últimos años se observa una reorientación en los presupuestos destinados a las comunicaciones de marketing hacia los denominados medios alternativos (entre los que se incluye el emplazamiento de producto, el marketing de eventos, el marketing móvil o la publicidad en videojuegos). Esta tendencia queda bien reflejada en el crecimiento a nivel mundial de la inversión en medios alternativos durante los últimos años, con un promedio del 21,7\% desde 2002 hasta 2007 (PQ Media, 2008). En este sentido, resulta significativo que una marca de referencia como CocaCola anunciara hace años que iba a incrementar sus recursos publicitarios en videojuegos y DVD (Grover et al. 2004). En especial, en los últimos años está adquiriendo un creciente protagonismo el denominado branded entertainment que consiste en la hibridación de contenido publicitario y contenido de entretenimiento como películas o videojuegos. Se trata de una estrategia que se desarrolla en un contexto de creciente convergencia entre los medios de comunicación y los productores de contenido (Donaton, 2004) para la que se ha estimado un crecimiento de inversión, a nivel mundial, en los próximos años de en torno al 9.1\% hasta el 2014 y en los que el advergaming (la hibridación de contenido publicitario y videojuegos) ocupa un lugar destacado (PQ Media, 2010).
Este interés en la praxis comercial ha ido acompañado en los últimos años por un creciente interés de la comunidad académica por entender mejor el funcionamiento de este tipo de herramientas publicitarias, sus efectos sobre diferentes parámetros de interés para el marketing (como por ejemplo, los efectos sobre la memoria y las actitudes) y, en definitiva, sobre cómo conocer mejor, optimizar y hacer más efectivas este tipo de comunicaciones de marketing. Así en los últimos años, en el contexto de la publicidad mediante videojuegos, se han realizado trabajos de los efectos sobre la memoria de las marcas/productos emplazados en videojuegos (Grigorovici y Constantin, 2004; Nelson, 2002; Schneider y Cornwell, 2005; Yang et al. 2006); sobre los perfiles psicográficos de los jugadores online (Youn, Lee y Doyle, 2003); sobre las actitudes hacia la publicidad en los videojuegos (Nelson, Keum y Yaros, 2004; Lehu, 2007; Winkler y Buckner, 2006); sobre las actitudes hacia el anuncio (Schneider y Cornwell, 2005); sobre los efectos en la intención de compra (Chaney, Lin y Chaney, 2004); sobre los efectos en la preferencia de marca (Grigorovici y Constantin, 2004; Mallinckrodt y Mizerski, 2007); la influencia de los videojuegos en los comportamientos de los consumidores (Molesworth, 2006); la actitud hacia los advergames (Hernández et al., 2004; Winkler y Buckner, 2006); y sobre el uso de este tipo de estrategias por parte de los anunciantes (Moore, 2006).

En este contexto, el presente trabajo exploratorio tiene como objetivo principal realizar una revisión de la literatura académica sobre la eficacia del uso de los videojuegos como herramienta publicitaria. Asimismo esta revisión debe conducir a la identificación de las principales líneas de investigación, factores y metodologías em- 
pleadas hasta la fecha. Finalmente debe permitirnos detectar áreas de estudio poco exploradas que posibilite apuntar futuras líneas de investigación de especial interés.

El presente trabajo exploratorio utiliza el análisis de contenido (Fearing, 1953; Ferber y Wales en 1958; Kassarjian, 1977) como herramienta de análisis. Esta herramienta ya ha sido utilizada anteriormente para el análisis de artículos publicados en revistas académicas (Hirschman, 1991). El análisis de contenido cuenta con una larga tradición como herramienta de análisis de los mensajes emitidos por los medios de comunicación y ya ha sido aplicado con éxito para detectar cómo son integradas las marcas y mensajes publicitarios en los videojuegos (Moore, 2006).

El presente trabajo se estructura de la siguiente manera: en primer lugar se realiza una contextualización del objeto de estudio (la publicidad en videojuegos). Este primer apartado tiene como objetivo ofrecer una breve aproximación histórica al uso de los videojuegos como herramienta publicitaria así como conceptualizar las principales tipologías utilizadas. En el siguiente apartado se ofrece la metodología utilizada y se presenta tanto la ficha de análisis como la muestra utilizada. A continuación se procede al análisis de los resultados. Finalmente se exponen las conclusiones derivadas de este análisis.

\section{La publicidad en videojuegos}

El uso de los videojuegos como herramienta publicitaria ha experimentado un importante crecimiento en los últimos años. Este fenómeno queda reflejado en el hecho de que las principales agencias de medios y de publicidad hayan abierto en los últimos años divisiones especializadas en advergaming o en emplazamiento publicitario en videojuegos (por ejemplo, The Bounce Interac- tive Gaming Group -BIG-, perteneciente a Young \& Rubicam, o Play Division de Starcom Media). La compra de Massive en 2006 por parte de Microsoft o de Adscape por Google en 2007 (ambas empresas especializadas en la inserción de anuncios en tiempo real en videojuegos online) también demuestra el interés de los gigantes de las nuevas tecnologías por entrar en un mercado para el que la inversión publicitaria ha sido estimada en torno a los mil millones de dólares en 2012 (eMarketer, 2008).

A pesar de este incremento en los últimos años de la utilización de los videojuegos como herramienta publicitaria su uso no es algo nuevo. Más bien lo contrario, se ha sugerido que surge con el nacimiento mismo de los videojuegos como medio de entretenimiento de masas. Vedrashko (2006) sitúa el inicio de un verdadero interés de los anunciantes por utilizar los videojuegos como herramienta publicitaria en torno a 1982, coincidiendo con el primer despegue de la industria de los videojuegos. Ese año el público norteamericano se gastó más de 3.000 millones de dólares en videojuegos triplicando la cantidad invertida el año anterior (De Meyer, Malliet y Verbruggen, 2001, cfr. en Vedrashko, 2006). Así, Budweiser emplazaba en 1984 su marca en el gran éxito del año, Tapper (Bally/Midway, 1983), e incluso McDonald’s desarrolló en 1983 un pionero videojuego con Ronald McDonald's como protagonista. Desde entonces el incremento de la presencia de marcas o mensajes publicitarios en videojuegos ha sido constante. Por citar sólo unos cuantos ejemplos, Pepsi emplazó su refresco en el juego Fighting Vipers (Sega: 1995); Coca-Cola hizo lo propio al emplazar una de sus máquinas expendedoras de refrescos en el gran éxito de 1998 Half-Life (Sierra, 1998); el mismo año, Yamaha, Michelin y Mobil (entre otras mar- 
cas) emplazaron sus respectivas marcas en Moto Racer 2 (Electronic Arts, 1998). Ya más recientemente, Dole Food Company emplazó pegatinas suyas sobre las bananas que aparecían en Super Monkey Ball (Sega: 2001), haciéndolo coincidir con el momento en el que lanzaba una nueva línea de bananas de lujo en el mercado japonés (Emery, 2002). El mundo de la moda tampoco ha permanecido al margen de este tipo de iniciativas y tanto Paco Rabanne como Calvin Klein (junto a otros anunciantes como Samsung) emplazaron su publicidad en la futurista ciudad de New York Race (Wanadoo Edition, 2001). Otros casos también muy conocidos son los de Pizza Hut y KFC, que poblaron con sus restaurantes de comida rápida los escenarios de Crazy Taxi (Acclaim, 2001). En la actualidad es habitual la presencia de cada vez más marcas en los escenarios de los videojuegos, sobre todo en los más vendidos, debido a su alcance cada vez mayor sobre diferentes audiencias y consumidores. De hecho, el interés de los anunciantes por utilizar los videojuegos como herramienta publicitaria ha crecido a la par que han aumentado y diversificado sus audiencias. Si hasta hace apenas 15 años el consumidor mayoritario de videojuegos era varón de entre 18-35 años, en la actualidad se puede afirmar que desde hace unos años los videojuegos son consumidos virtualmente por cualquier segmento de edad de ambos sexos (Fattah y Paul, 2002).

\subsection{Tipologías publicitarias en videojuegos}

Las principales tipologías mediante las cuales los videojuegos son utilizados como herramienta publicitaria son el emplazamiento de producto, el in-game advertising y el advergaming. El emplazamiento de producto ha sido definido como "la práctica de incluir el nombre, producto, embala- je, señalización u otro artículo de merchandising de una marca, en una película de cine, en televisión o en otros medios con el fin de incrementar la notoriedad y el reconocimiento inmediato de la marca en el punto de compra" (Panda, 2004, p. 7). El primer emplazamiento de producto en videojuegos que se conoce parece ser el que realizó Scott Adams en su propio videojuego Adventureland (1978) anunciando su próximo videojuego Pirate Adventure. El emplazamiento de producto en videojuegos se realiza mediante la inclusión de pancartas, anuncios o los propios productos en los escenarios de juego. Generalmente se suele diferenciar entre emplazamientos on-set (suelen ser objetos tridimensionales emplazados en primer plano con los que generalmente puede interactuar el videojugador), y emplazamientos periféricos o creativos (anuncios bidimensionales como vallas, carteles luminosos, etc. emplazados como atrezzo del espacio de juego) en función de la prominencia del emplazamiento.

El in-game advertising consiste en el emplazamiento de anuncios dinámicos en los escenarios de los videojuegos online. Esta técnica permite el emplazamiento en tiempo real de anuncios que se ajusten a los perfiles sociodemográficos de los jugadores online. Por ejemplo, en el videojuego online SWAT 4 (Vivendi Universal Games, 2005), la empresa Massive emplazó (sólo para los videojugadores norteamericanos) anuncios de programas televisivosl cuyo estreno era inminente en los EE.UU. Es decir, estos anuncios dinámicos online permiten desarrollar campañas en las que el timing es importante (estrenos cinematográficos o televisivos, fechas de eventos con los que se haga coincidir la campaña, etc.) e incluso realizar

1 Por ejemplo, la serie Lust in Space que se iba a estrenar en el canal SciFi. 
rotaciones de los anuncios emplazados con respecto a la estacionalidad (campañas de navidad o verano, rebajas, etc.).

Finalmente, el advergaming consiste en el desarrollo ex profeso de videojuegos orientados a las comunicaciones de marketing (Martí, 2010). Grundy (2008) afirma que el primer advergame de la historia fue probablemente Datsun 280 Zzzap, un videojuego desarrollado en 1976 para promocionar el Datsun 280 Z. Otros de estos primeros advergames fueron Tooth Protectors (creado en 1982 por Johnson \& Johnson), Chase the Chuckwagon (creado también en 1982 por Ralston-Purina), y Pepsi Invaders. Este último advergame, basado en Space Invaders (Taito, 1978), fue desarrollado por Atari en 1983 para CocaCola y regalado a los asistentes a una convención de ventas en Atlanta. Los alienígenas del videojuego original fueron reemplazados por las letras P. E. P. S. I. y la nave comandante que aparecía en la parte superior de la pantalla mostraba el logotipo de Pepsi. Al terminar los tres minutos de límite de juego aparecía un mensaje parpadeante en la pantalla con la leyenda "Coca-Cola Gana".

El aumento en la diversificación de las herramientas para desarrollar comunicaciones publicitarias mediante videojuegos así como una mayor sofisticación y creciente complejidad de este tipo de acciones ha conducido a un encarecimiento de este tipo de acciones. Así, los costes de emplazamiento publicitario en videojuegos que alcanzan audiencias millonarias (los denominados AAA) se han cifrado en una horquilla que abarca entre los 25.000 y los 700.000 dólares en función de la prominencia y el grado de integración en la trama de la marca o el producto emplazado (Moran, 2004). Asimismo, el coste medio del desarrollo de un advergame oscila en- tre los 10.000 y 35.000 dólares para los advergames sindicados (IGDA, 2005) aunque esta cantidad puede alcanzar o superar los 500.000 dólares para otro tipo de advergames. De forma similar, también han aumentado las expectativas de los anunciantes a la hora de conocer el retorno de su inversión en publicidad en videojuegos. Las métricas más comunes actualmente utilizadas incluyen no sólo métricas tradicionales utilizadas para medir la eficacia de la publicidad en videojuegos, como las tasas de recuerdo, sino los cambios en el reconocimiento y preferencia de marca, los cambios en el comportamiento del consumidor, o la frecuencia y duración de las sesiones de juego (IGDA, 2008).

\section{Metodología de estudio}

Dados los objetivos del presente trabajo se optó por el uso del análisis de contenido (Fearing, 1953; Ferber y Wales, 1958; Kassarjian, 1977). Esta herramienta de análisis consiste en "una técnica de investigación por medio de la descripción objetiva, sistemática y cuantitativa del contenido manifiesto de un comunicador" (Berelson, 1952, p. 55). Entre las ventajas que ofrece esta herramienta se encuentra la uniformidad del análisis efectuado por diferentes investigadores, la disminución de los juicios personales y subjetivos en el procesamiento de la información y el análisis de los contenidos tanto latentes como manifiestos en base a unos criterios objetivamente definidos por los jueces que efectúan el análisis (Fearing, 1953). Este análisis permite no sólo la transformación de la información en datos que pueden ser resumidos y comparados (Paisley, 1969) sino que permiten extraer información que debe estar vinculada a otra información con la que explicar algún hecho (Berelson, 1952). 


\subsection{Desarrollo de la herramienta de análisis}

Como herramienta de análisis se desarrolló una ficha con descriptores correspondientes a cada una de las dimensiones de análisis planteadas. Así, esta ficha recoge un apartado referido a la información general de cada artículo compuesto por los siguientes descriptores: año de publicación, título del artículo, autores y revista en la que se publicó el artículo. Una segunda dimensión hace referencia a la metodología de estudio empleada en cada artículo. Los descriptores incluidos en este apartado fueron: artículo (identificado por el nombre del autor/es), tipología de estudio (cuantitativo/cualitativo), herramienta de análisis (análisis factorial, modelo de ecuaciones estructurales, etc.), tamaño muestral y factores analizados en el artículo (congruencia, intrusión, entretenimiento, etc.). Una última dimensión analizaba los efectos sobre el consumidor estudiados en cada artículo. Estos efectos fueron clasificados como cognitivos (recuerdo, reconocimiento...), afectivos (actitud hacia la marca, identificación con la marca...) y conativos (elección de marca, intención de compra...), siguiendo el Modelo de la Jerarquía de los Efectos (Lavidge y Steiner, 1961), y son habitualmente utilizados como parámetro de medida de eficacia tanto en las comunicaciones publicitarias como en otras acciones de las comunicaciones de marketing. La herramienta de análisis se recoge en la tabla 1.

\subsection{Muestra}

Con el fin de seleccionar una muestra de trabajos académicos representativos del tema a analizar se procedió a realizar una búsqueda en una base de datos especializada de revistas académicas (ProQuest) en torno a palabras clave relacionadas con el objeto de estudio (publicidad y vi-

\section{Tabla $1 \cdot$ Herramienta de análisis}

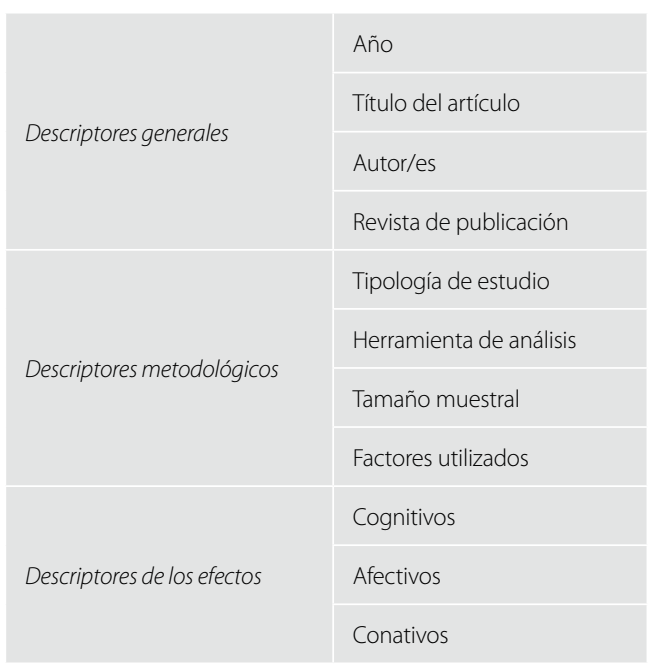

deojuegos). Tras la búsqueda en la base de datos se identificaron 19 trabajos cuyos títulos y resúmenes se correspondían con el objeto de estudio (tabla 2 - pág. 27).

\section{Análisis de los resultados}

El análisis de los resultados muestra un uso predominante de la investigación cuantitativa frente a la cualitativa. Sólo tres de los 19 artículos analizados incluía algún tipo de análisis cualitativo a la hora de medir la eficacia de las acciones publicitarias en videojuegos. Asimismo predominan los estudios experimentales en los que la herramienta de análisis principal es el análisis factorial (10). Otros tipos de análisis utilizados incluyen los análisis descriptivos (4) que incluían tanto análisis de correlación como test-t. Tan sólo un estudio utiliza el focus group como herramienta de análisis mientas que otro utiliza herramientas webnográficas ${ }^{2}$ y otro un modelo de ecuaciones estructurales. En esta misma situación se encuentran los análisis multivariantes (MANOVA) y bi-

2 Kozinets (2002). 


\section{Tabla 2 - Descriptores generales de los artículos}

\begin{tabular}{|c|c|c|c|}
\hline Año & Título & Autor & Publicación \\
\hline 2002 & Recall of Brand Placements in Computer/Video Games & Nelson & $\begin{array}{l}\text { Journal of Advertising } \\
\text { Research }\end{array}$ \\
\hline 2003 & Lifestyles of Online Gamers: A Psychographic Approach & Youn, Lee y Doyle & $\begin{array}{l}\text { Journal of Interactive } \\
\text { Advertising }\end{array}$ \\
\hline 2004 & The Effect of Billboards within the Gaming Environment & Chaney, Lin y Chaney & $\begin{array}{l}\text { Journal of Interactive } \\
\text { Advertising }\end{array}$ \\
\hline 2004 & $\begin{array}{l}\text { Advertainment or Adcreep? Game Players' Attitudes toward } \\
\text { Advertising and Product Placements in Computer Games }\end{array}$ & Nelson, Keum y Yaros & $\begin{array}{l}\text { Journal of Interactive } \\
\text { Advertising }\end{array}$ \\
\hline 2004 & $\begin{array}{l}\text { Hispanic Attitudes toward Advergames: A Proposed Model } \\
\text { of their Antecedents }\end{array}$ & $\begin{array}{l}\text { Hernández, Chapa, Minor, } \\
\text { Maldonado y Barranzuela }\end{array}$ & $\begin{array}{l}\text { Journal of Interactive } \\
\text { Advertising }\end{array}$ \\
\hline 2004 & $\begin{array}{l}\text { Experiencing Interactive Advertising Beyond Rich Media: } \\
\text { Impacts of Ad Type and Presence on Brand Effectiveness } \\
\text { in 3D Gaming Immersive Virtual Environments }\end{array}$ & Grigorovici y Constantin & $\begin{array}{l}\text { Journal of Interactive } \\
\text { Advertising }\end{array}$ \\
\hline 2005 & $\begin{array}{l}\text { Cashing in on crashes via brand placement in computer } \\
\text { games: The effects of experience and flow on memory }\end{array}$ & Schneider y Cornwell & $\begin{array}{l}\text { International Journal } \\
\text { of Advertising }\end{array}$ \\
\hline 2005 & $\begin{array}{l}\text { The Effect of Involvement on Ad Judgment in a Video Game } \\
\text { Environment: The Mediating Role of Presence }\end{array}$ & Nicovich & $\begin{array}{l}\text { Journal of Interactive } \\
\text { Advertising }\end{array}$ \\
\hline 2006 & $\begin{array}{l}\text { Examining The Influence Of Telepresence On Spectator And } \\
\text { Player Processing Of Real and Fictitious Brands in a Computer } \\
\text { Game }\end{array}$ & Nelson, Yaros y Deum & Journal of Advertising \\
\hline 2006 & $\begin{array}{l}\text { Receptiveness of Gamers to Embedded Brand Messages } \\
\text { in Advergames: Attitudes towards Product Placement }\end{array}$ & Winkler y Buckner & $\begin{array}{l}\text { Journal of Interactive } \\
\text { Advertising }\end{array}$ \\
\hline 2006 & $\begin{array}{l}\text { Real Brands in Imaginary Worlds: Investigating Players' Experi- } \\
\text { ences of Brand Placement in Digital Games }\end{array}$ & Molesworth & $\begin{array}{l}\text { Journal of Consumer } \\
\text { Behaviour }\end{array}$ \\
\hline 2006 & $\begin{array}{l}\text { The Effectiveness of 'in-game' advertising: Comparing Collage } \\
\text { Students' Explicit and Implicit Memory for Brand Names }\end{array}$ & $\begin{array}{l}\text { Yang, Roskos-Ewoldsen, } \\
\text { Dinu y Arpan }\end{array}$ & Journal of Advertising \\
\hline 2007 & $\begin{array}{l}\text { Effects of Product Placement in On-Line Games on Brand } \\
\text { Memory: A Perspective of the Limited Capacity Model of } \\
\text { Attention }\end{array}$ & Lee y Faber & Journal of Advertising \\
\hline 2007 & $\begin{array}{l}\text { The Effects of Playing an Advergame on Young Children's } \\
\text { Perceptions, Preferences, and Requests }\end{array}$ & Mallinckrodt y Mizerski & Journal of Advertising \\
\hline 2007 & The Effectiveness of Product Placement in Video Games & Glass & $\begin{array}{l}\text { Journal of Interactive } \\
\text { Advertising }\end{array}$ \\
\hline 2008 & $\begin{array}{l}\text { Enjoyment of Advergames and Brand Attitudes: The Impact } \\
\text { of Thematic Relevance }\end{array}$ & $\begin{array}{l}\text { Wise, Bolls, Kim, Venkata- } \\
\text { raman, y Meyer }\end{array}$ & $\begin{array}{l}\text { Journal of Interactive } \\
\text { Advertising }\end{array}$ \\
\hline 2010 & $\begin{array}{l}\text { Advergames: The Impact of Brand Prominence and Game } \\
\text { Repetition on Brand Responses }\end{array}$ & $\begin{array}{l}\text { Cauberghe y De Pels- } \\
\text { macker }\end{array}$ & Journal of Advertising \\
\hline 2010 & $\begin{array}{l}\text { In-Game Advertising Effects: Examining Player Perceptions } \\
\text { Of Advertising Schema Congruity In A Massively Multiplayer } \\
\text { Online Role-Playing Game }\end{array}$ & Lewis y Porter & $\begin{array}{l}\text { Journal of Interactive } \\
\text { Advertising }\end{array}$ \\
\hline 2010 & $\begin{array}{l}\text { Online In-Game Advertising Effect: Examining The Influence } \\
\text { Of A Match Between Games And Advertising }\end{array}$ & Chang, Yan, Zhang y Luo & $\begin{array}{l}\text { Journal of Interactive } \\
\text { Advertising }\end{array}$ \\
\hline
\end{tabular}


variantes (ANOVA) con un estudio cada uno. Por lo que respecta a los tamaños muestrales utilizados en los estudios resultan bastante heterogéneos: siete de los artículos analizados utilizaron una muestra de entre 16-50 observaciones: dos artículos utilizaron una muestra de 62 observaciones; cinco artículos utilizaron una muestra en torno a las 100 y 150 observaciones mientras que los cinco artículos restantes excedieron notablemente estas cantidades (con 295, 443, 480, 805 y 3056 observaciones respectivamente). Con respecto a los factores utilizados como variables de análisis en los artículos, tres artículos contemplan el tipo de emplazamiento de las marcas o productos en el videojuego (bien el espacio del videojuego en el que tiene lugar el emplazamiento o el tamaño del propio emplazamiento) así como el género de videojuego uti- lizado (mayoritariamente videojuegos de carreras o deportivos), la congruencia de las marcas emplazadas y la implicación del videojugador con el videojuego; dos artículos contemplan la experiencia de juego del videojugador (experiencia previa como videojugador) y el tipo de marca emplazada (real o ficticia) así como el sentimiento de presencia que el videojugador es capaz de experimentar mientras está jugando. Otras variables presentes en los estudios son: el estado de flujo; las características demográficas, motivacionales y actitudinales de los sujetos; la categoría de producto emplazado; la valencia de las opiniones; la intrusión; el tiempo de exposición a la marca; la irritación; el entretenimiento; el contexto de uso (si el sujeto juega o ve el videojuego); y el tipo de memoria analizada (implícita y explícita).

\section{Tabla 3 - Efectos analizados en los artículos}

\begin{tabular}{|c|c|c|c|}
\hline Artículo & Efectos cognitivos & Efectos afectivos & Efectos Conativos \\
\hline Nelson (2002) & Recuerdo de la marca emplazada & Actitud hacia el emplazamiento & \\
\hline Youn, Lee y Doyle (2003) & & Actitud hacia la publicidad & \\
\hline Chaney, Lin y Chaney (2004) & Recuerdo de la marca emplazada & Actitud hacia el emplazamiento & Intención de compra \\
\hline Nelson, Keum y Yaros (2004) & & $\begin{array}{l}\text { Actitud hacia el emplazamiento. } \\
\text { Actitud hacia la publicidad }\end{array}$ & Intención de compra \\
\hline Hernández et al. (2004) & & Actitud hacia el advergame & \\
\hline $\begin{array}{l}\text { Grigorovici y Constantin } \\
\text { (2004) }\end{array}$ & $\begin{array}{l}\text { Recuerdo y reconocimiento de la } \\
\text { marca/producto emplazado }\end{array}$ & Preferencia de marca & \\
\hline Schneider y Cornwell (2005) & $\begin{array}{l}\text { Recuerdo y reconocimiento de la } \\
\text { marca emplazada }\end{array}$ & & \\
\hline Nicovich (2005) & & Actitud hacia el emplazamiento & \\
\hline Nelson, Yaros y Keum (2006) & Recuerdo de la marca emplazada & Actitud hacia el emplazamiento & \\
\hline Winkler y Buckner (2006) & Recuerdo de la marca emplazada & Actitud hacia el emplazamiento & \\
\hline Molesworth (2006) & Recuerdo de la marca emplazada & & Comportamiento de uso \\
\hline
\end{tabular}




\begin{tabular}{|c|c|c|c|}
\hline Artículo & Efectos cognitivos & Efectos afectivos & Efectos Conativos \\
\hline Yang et al. (2006) & $\begin{array}{l}\text { Recuerdo y reconocimiento de la } \\
\text { marca emplazada }\end{array}$ & & \\
\hline Lee y Faber (2007) & $\begin{array}{l}\text { Recuerdo y reconocimiento de la } \\
\text { marca emplazada }\end{array}$ & & \\
\hline $\begin{array}{l}\text { Mallinckrodt y Mizerski } \\
\text { (2007) }\end{array}$ & & Preferencia de marca & \\
\hline Glass (2007) & & Actitud hacia la marca & \\
\hline Wise et al. (2008) & & $\begin{array}{l}\text { Actitud hacia el advergame. } \\
\text { Actitud hacia la marca }\end{array}$ & \\
\hline $\begin{array}{l}\text { Cauberghe y De Pelsmacker } \\
\text { (2010) }\end{array}$ & $\begin{array}{l}\text { Recuerdo de la marca } \\
\text { emplazada }\end{array}$ & Actitud hacia la marca & \\
\hline Lewis y Porter (2010) & $\begin{array}{l}\text { Notoriedad de la marca } \\
\text { emplazada }\end{array}$ & Actitud hacia el emplazamiento & \\
\hline $\begin{array}{l}\text { Chang, Yan, Zhang y Luo } \\
\text { (2010) }\end{array}$ & & & Intención de compra \\
\hline
\end{tabular}

Con respecto a los efectos analizados, 11 de los trabajos miden la eficacia publicitaria en relación a efectos cognitivos (principalmente recuerdo y reconocimiento de las marcas o productos emplazados) mientras que 10 de los trabajos miden la eficacia publicitaria en relación a las respuestas afectivas de los usuarios (principalmente actitud hacia la marca o hacia el emplazamiento). Sólo 2 de estos 10 trabajos miden los efectos afectivos en relación a la preferencia mostrada por la marca. Cabe destacar que 7 de los trabajos analizados miden la eficacia publicitaria en relación a efectos tanto cognitivos como afectivos. Tan sólo 3 de los artículos analizados miden la eficacia publicitaria en relación a efectos conativos (tres en relación a la intención de compra y uno de ellos, además, en relación al comportamiento de uso de los productos emplazados).

\section{Tabla 4 • Metodología empleada en los artículos}

\begin{tabular}{|c|c|c|c|c|c|}
\hline \multirow{2}{*}{ Artículo } & \multicolumn{2}{|c|}{ Tipología de estudio } & \multirow{2}{*}{ Herramienta de análisis } & \multirow{2}{*}{$\begin{array}{l}\text { Tamaño } \\
\text { muestral }\end{array}$} & \multirow{2}{*}{ Factores } \\
\hline & Cuanti. & Cuali. & & & \\
\hline Nelson (2002) & $x$ & $x$ & $\begin{array}{l}\text { Análisis factorial } \\
\text { Preguntas abiertas }\end{array}$ & 16 & Tipo de marca (real/ficticia) \\
\hline $\begin{array}{l}\text { Youn, Lee y Doyle } \\
\text { (2003) }\end{array}$ & & & Análisis factorial & 3056 & $\begin{array}{l}\text { Características demográficas (usuari- } \\
\text { os/ no usuarios de internet, edad e } \\
\text { ingresos), motivacionales (impulsivi- } \\
\text { dad, búsqueda de variedad/riesgo, } \\
\text { búsqueda de diversión y boca a } \\
\text { boca) y actitudinales (liberalismo, } \\
\text { actitud hacia la publicidad y actitud } \\
\text { hacia internet).. }\end{array}$ \\
\hline $\begin{array}{l}\text { Chaney, Lin y } \\
\text { Chaney (2004) }\end{array}$ & $x$ & & $\begin{array}{l}\text { Análisis descriptivo univariado } \\
\text { Prueba } \mathrm{C}_{2} \\
\text { Correlaciones }\end{array}$ & 42 & $\begin{array}{l}\text { Experiencia de juego. } \\
\text { Categoría de producto. }\end{array}$ \\
\hline
\end{tabular}




\begin{tabular}{|c|c|c|c|c|c|}
\hline \multirow{2}{*}{ Artículo } & \multicolumn{2}{|c|}{ Tipología de estudio } & \multirow{2}{*}{ Herramienta de análisis } & \multirow{2}{*}{$\begin{array}{l}\text { Tamaño } \\
\text { muestral }\end{array}$} & \multirow{2}{*}{ Factores } \\
\hline & Cuanti. & Cuali. & & & \\
\hline $\begin{array}{l}\text { Nelson, Keum y } \\
\text { Yaros (2004) }\end{array}$ & $x$ & $x$ & $\begin{array}{l}\text { Técnicas webnográficas } \\
\text { Análisis de regresión }\end{array}$ & $\begin{array}{l}805 \text { posts. } \\
62\end{array}$ & $\begin{array}{l}\text { Valencia de las opiniones (positiva, } \\
\text { negativa o neutra). }\end{array}$ \\
\hline $\begin{array}{l}\text { Hernández et } \\
\text { al.(2004) }\end{array}$ & $x$ & & $\begin{array}{l}\text { Modelo de ecuaciones } \\
\text { estructurales }\end{array}$ & $98 / 126 / 91$ & $\begin{array}{l}\text { Congruencia, intrusión, tiempo } \\
\text { de exposición, entretenimiento e } \\
\text { irritación. } \\
\text { Género de videojuego. }\end{array}$ \\
\hline $\begin{array}{l}\text { Grigorovici y Con- } \\
\text { stantin (2004) }\end{array}$ & $x$ & & Análisis factorial & 144 & $\begin{array}{l}\text { Tipo de emplazamiento (on-set, } \\
\text { creativo). } \\
\text { Implicación con el videojuego (alta/ } \\
\text { baja). }\end{array}$ \\
\hline $\begin{array}{l}\text { Schneider y Corn- } \\
\text { well (2005) }\end{array}$ & $x$ & & Análisis factorial & 46 & $\begin{array}{l}\text { Prominencia espacial del emplaza- } \\
\text { miento (alta/baja). } \\
\text { Prominencia en el juego (alta/baja). } \\
\text { Estado de flujo. } \\
\text { Experiencia de juego. }\end{array}$ \\
\hline Nicovich (2005) & $x$ & & Análisis factorial & 152 & $\begin{array}{l}\text { Implicación } \\
\text { Presencia. }\end{array}$ \\
\hline $\begin{array}{l}\text { Nelson, Yaros y } \\
\text { Keum (2006) }\end{array}$ & $x$ & & Análisis factorial & 62 & $\begin{array}{l}\text { Contexto del medio (jugado/ } \\
\text { visionado). } \\
\text { Tipo de marca (real/ficticia) } \\
\text { Telepresencia. }\end{array}$ \\
\hline $\begin{array}{l}\text { Winkler y Buckner } \\
\text { (2006) }\end{array}$ & $x$ & & Análisis descriptivo univariado & 42 & $\begin{array}{l}\text { Tipo de advergame (asociativo, } \\
\text { ilustrativo y demostrativo). } \\
\text { Género de de videojuego. }\end{array}$ \\
\hline Molesworth (2006) & & $x$ & Focus groups & 30 & Experiencias de juego. \\
\hline Yang et al.(2006) & $x$ & & Análisis factorial & 153 & $\begin{array}{l}\text { Género de videojuego } \\
\text { Tipo de memoria (implícita y ex- } \\
\text { plícita). }\end{array}$ \\
\hline Lee y Faber (2007) & $x$ & & $\begin{array}{l}\text { Análisis multivariante } \\
\text { (MANOVA) }\end{array}$ & 155 & $\begin{array}{l}\text { Proximidad (focal/periférica). } \\
\text { Congruencia } \\
\text { Implicación con el videojuego (alta/ } \\
\text { moderada) } \\
\text { Experiencia de juego. }\end{array}$ \\
\hline $\begin{array}{l}\text { Mallinckrodt y Miz- } \\
\text { erski (2007) }\end{array}$ & $x$ & & Análisis descriptivo univariado & 295 & $\begin{array}{l}\text { Preferencia hacia la marca } \\
\text { Conocimiento de la persuasión }\end{array}$ \\
\hline Glass (2007) & $x$ & & $\begin{array}{l}\text { Análisis descriptivo univariado } \\
\text { Test } t \text { muestras independientes }\end{array}$ & 28 & $\begin{array}{l}\text { Tipo de memoria (implícita y ex- } \\
\text { plícita). }\end{array}$ \\
\hline Wise et al. (2008) & $x$ & & Análisis factorial & 43 & $\begin{array}{l}\text { Conexión temática (congruencia). } \\
\text { Género de videojuego. }\end{array}$ \\
\hline $\begin{array}{l}\text { Cauberghe y De } \\
\text { Pelsmacker (2010) }\end{array}$ & $x$ & & Análisis factorial & 480 & $\begin{array}{l}\text { Prominencia, número de veces que } \\
\text { el juego es jugado e implicación } \\
\text { con el producto. }\end{array}$ \\
\hline $\begin{array}{l}\text { Lewis y Porter } \\
\text { (2010) }\end{array}$ & $x$ & & $\begin{array}{l}\text { Análisis descriptivo univariado } \\
\text { Test ANOVA }\end{array}$ & 100 & Congruencia \\
\hline $\begin{array}{l}\text { Chang, Luo, Yan y } \\
\text { Zhang (2010) }\end{array}$ & $x$ & & Análisis factorial & 443 & $\begin{array}{l}\text { Congruencia, integración, promi- } \\
\text { nencia, interés. }\end{array}$ \\
\hline
\end{tabular}




\section{Conclusiones}

El análisis metodológico muestra un dominio de los estudios cuantitativos a la hora de medir la eficacia de las acciones publicitarias en videojuegos. Dentro de estos estudios son también mayoritarios los estudios experimentales en condiciones de laboratorio. Se observa pues una carencia de estudios experimentales u otro tipo de estudios empíricos en contextos de uso reales (sólo tres de los estudios se realizaron en contextos online que simulaban situaciones reales en las que los usuarios podían encontrarse y jugar a videojuegos con contenidos publicitarios). En este sentido resulta necesario aumentar nuestro conocimiento sobre el rendimiento de teorías y modelos que han sido testados en pruebas de laboratorio en contextos de uso reales. También se observa un limitado uso de herramientas cualitativas (como los focus group) en el estudio de la eficacia de las acciones publicitarias en videojuegos.

Los tamaños muestrales de los artículos son bastante heterogéneos aunque existe una predominancia de trabajos (nueve) en los que la muestra es menor de 100 observaciones. Esto es debido fundamentalmente a la naturaleza experimental de los artículos cuyas condiciones de laboratorio dificulta trabajar con muestras excesivamente grandes. El pequeño tamaño muestral de algunos artículos (y la falta de representatividad de la muestra) presenta limitaciones como la de poder extrapolar las conclusiones obtenidas a segmentos de la población. Futuras investigaciones deberían trabajar con muestras representativas que permitieran extrapolar los resultados al conjunto de la población.

El análisis de los resultados permite identificar claramente líneas de investigación emergentes a la hora de medir la eficacia de las acciones publicitarias en videojuegos. Así, factores de dise- ño (Martí, 2010) como el tipo de emplazamiento (en relación a variables como la proximidad), el género de videojuego utilizado o la congruencia de las marcas/productos en relación a los géneros de videojuegos son comunes a diversos de los artículos analizados. También se ha detectado un interés recurrente en torno a factores individuales (Martí, 2010) como la implicación del videojugador con el género de videojuego, y la experiencia de juego. Por otra parte, sólo un estudio trabaja con un factor como el contexto del medio (si el usuario está jugando o viendo cómo otras personas juegan al videojuego). Los aficionados a los videojuegos no sólo juegan a ellos sino que pueden ver cómo otros jugadores lo hacen. Los campeonatos mundiales de videojuegos (como los World Cyber Games o los Electronic Sports World Cup) congregan a miles de aficionados de todo el mundo que asisten a este tipo de competiciones en las que las partidas son proyectadas en pantallas gigantes convirtiendo en audiencia cautiva a los asistentes ${ }^{3}$. El incremento de espacios televisivos o sitios web que ofrecen información específica sobre videojuegos (por ejemplo, tráileres) también posibilita una audiencia de espectadores expuestos a mensajes publicitarios en videojuegos bajo esta condición. En este sentido parece necesario incrementar el conocimiento sobre los efectos de la publicidad en videojuegos no sólo cuando el consumidor los juega sino también cuando los ve. Resulta también llamativo que un factor individual como el entretenimiento percibido haya recibido una escasa atención académica. Tan sólo uno de los estudios contempla esta variable a pesar de que el

3 Según la organización del Máster de París de 2008 de los Electronic Sports World Cup, al evento acudieron 450 videojugadores de 20 países, con una afluencia total de público de 31.000 personas durante los 3 días que duró la competición. 
entretenimiento es considerado un motivo fundamental por el que los consumidores juegan a los videojuegos (Interactive Digital Software Association, 2000).

Ninguno de los estudios considera como factor de análisis la modalidad del emplazamiento de la marca en el videojuego (sonoro, visual o audiovisual). Sin embargo la hipótesis de la codificación redundante de Paivio (1979) ha sido testada con éxito en otros contextos de emplazamiento de producto como un contenido audiovisual que simulaba una serie de televisión. En este sentido, Russell (1999) detectó que los emplazamientos duales (tanto visuales como verbales) influyeron más sobre la memoria de los sujetos que los emplazamientos que utilizaron una sola modalidad (por ejemplo, los emplazamientos sólo verbales o los emplazamientos sólo visuales) ya que "el incremento en la disponibilidad de los dos códigos incrementa la probabilidad de recuerdo de un objeto porque la respuesta puede ser recuperada de cualquiera de los dos códigos" (Paivio, 1979, p. 297). Esta línea de investigación se presenta pues como una oportunidad para incrementar nuestro conocimiento en el campo de estudio. En base a los artículos analizados otros factores de diseño como las propias características del estímulo (por ejemplo un anuncio de la marca o del producto emplazado) como el color, el tamaño, el número de palabras, el tamaño de la imagen, o la presencia de animación, han sido escasamente estudiados con apenas un trabajo que haya abordado la cuestión (en este caso el tamaño). Por otra parte, a pesar de que se observa un interés por analizar los efectos del tipo de emplazamiento (en especial en relación con la prominencia) de marcas o productos en videojuegos poco o nada se sabe en el marco académico del modo en el que los anunciantes están emplazando mayoritariamente sus marcas o productos en videojuegos reales. En este sentido, el análisis de contenido de videojuegos reales que mostraran el modo en el que las marcas o productos son emplazados (vallas publicitarias, objetos, etc.) ayudaría a comprender mejor la praxis del mercado real. Del mismo modo un análisis de contenido en esta línea ayudaría a identificar el grado de saturación publicitaria presente en los videojuegos actuales (así como aquellos espacios o formatos más habitualmente utilizados).

A la luz de los resultados obtenidos se observa una carencia en el estudio de los efectos conativos de la publicidad en videojuegos. A pesar de la importancia de conocer los efectos cognitivos $\mathrm{y}$ afectivos de la publicidad en videojuegos (en especial la actitud hacia la marca como mediadora de los comportamientos de los consumidores), otros efectos conativos como la elección de marca parecen especialmente relevantes en los mercados actuales hipersaturados de mensaje publicitarios en los que comportamientos del consumidor como la compra impulsiva parecen primar el comportamiento del consumidor en función de los estímulos recibidos recientemente. En este sentido, puesto que los videojuegos pueden ser jugados cada vez en un mayor número de dispositivos portátiles (por ejemplo, en teléfonos móviles) conocer los efectos sobre la elección de marca provocados en un videojugador que puede encontrarse, por ejemplo, en un centro comercial parece especialmente relevante. Asimismo, puesto que el comercio electrónico pone a disposición de los consumidores una gran variedad de productos y servicios estos efectos deberían ser espacialmente analizados en aquellos videojugadores que juegan online. 


\section{Bibliografía}

Berelson, B. (1952). Content Analysis in Communications Research, Glencoe, Ill.: The Free Press

Cauberghe, V. y De Pelsmacker, P. (2010). "Advergames: The Impact of Brand Prominence and Game Repetition on Brand Responses", Journal of Advertising, 39(1), 5-18.

Chambers, J. (2006). "The Sponsored Avatar: Examining the Present Reality and Future Possibilities of Advertising in Digital Games,"

<http://ir.lib.sfu.ca/retrieve/1630/8878e0c3d9c0a0bc6767 0b8d9a0f.doc> (fecha de consulta: 04/05/07)

Chaney, I., Lin, K.H. y Chaney, J. (2004). "The Effect of Billboards within the Gaming Environment", Journal of Interactive Advertising, 5(1) <http://www.jiad.org/vol5/nol/chaney/> (fecha de consulta: 18/06/06)

Chang, Y., Yan, J., Zhang, J. y Luo, J. (2010). “Online InGame Advertising Effect: Examining The Influence Of A Match Between Games And Advertising", Journal of Interactive Advertising, 11(1), <http://jiad.org/article140> (fecha de consulta: 04/12/10)

Díaz Nosty, B. (2006). "Repensar la comunicación: la huella es el mensaje", en Tendencias '06 - Medios de Comunicación, Madrid: Fundación Telefónica, 15-43.

Donaton, S. (2004), Madison \& Vine: why the Entertainment and Advertising Industries Must Converge to Survive, Nueva York: McGraw-Hill.

eMarketer, 2008). http://www.emarketer.com/Reports/All/ Emarketer_2000485.aspx?src=report1_home

Emery, G. (2002). "Game Designers Go for Realism in Product Placements," <http://dailynews.yahoo.com> (fecha de consulta: 12/04/2006)

Fattah, H., y Paul, P. (2002). "Gaming Gets Serious," American Demographics, 24(5), 38-44.

Fearing, F. (1953) "Towards a Psychological Theory of Human Communication", Journal of Personality, 22, 71-88.

Ferber, R. y Wales, H.G. (ed.) (1958). Motivation and Market Behavior, Homewood, Ill.: Irwin.

Glass, Z. (2007). «The Effectiveness of Product Placement in Video Games», Journal of Interactive Advertising, 8(1), <http://jiad.org/article96> (fecha de consulta: 11/06/08)

Grigorovici, D.M. y Constantin, C.D. (2004). "Experiencing Interactive Advertising Beyond Rich Media: Impacts of Ad
Type and Presence on Brand Effectiveness in 3D Gaming Immersve Virtual Environments, Journal of Interactive Advertising, 5(1), <http://jiad.org/vol5/nol/grigorovici/index.htm> (fecha de consulta: 15/07/06)

Grover, R., Lowry, T., Khermouch, G., Edwards, C. y Foust, D. (2004). "Can Mad Ave. Make Zap-Proof Ads? It's Blurring The Lines Between Promotion and Programming As DVRs Gain Ground" Business Week, 3868 (2 de febrero), 36-37.

Grundy, J., (2009), Art History, en IGDA (2008).

Hernández, M. D., Chapa, S., Minor, M. S., Maldonado, C. y Barranzuela, F. (2004). "Hispanic Attitudes toward Advergames: A Proposed Model of their Antecedents", Journal of Interactive Advertising, 5(1), <http://jiad.org/vol5/nol/hernandez/index.htm> (fecha de consulta: 15/07/06)

Hirschman, E.C., (1991) "A feminist critique of marketing theory: toward agentic-communal balance", en Costa, J.A. (Ed.), Gender and Consumer Behaviour, University of Utah, Salt Lake City, UT, , pp. 324-40.

IGDA (International Games Developers Association) (2005). Casual Games White Paper, <http://www.igda.org/ casual/IGDA_CasualGames_Whitepaper_2005.pdf> (fecha de consulta: 20/06/07)

IGDA (International Games Developers Association) (2008). Casual Games White Paper, <http://www.igda.org/ casual/IGDA_CasualGames_Whitepaper_2008.pdf> (fecha de consulta: 12/04/09)

Interactive Digital Software Association (2002). Essential Facts about the Computer and the Video Game Industry.

Kassarjian, H. H. (1977). "Content Analysis in Consumer Research", Journal of Consumer Research, 4(1), 8-19.

Kozinets, R. V. (2002). "The Field Behind the Screen: Using Netnography for Marketing Research in Online Communities," Journal of Marketing Research, 39 (February), 61-72.

Lavidge, R. J. y Steiner, G. A. (1961). "A Model for Predictive Measurements of Advertising Effectiveness", Journal of Marketing, 24, 59-62.

Lee, M. y Faber, R.J. (2007). «Effects of Product Placement in On-Line Games on Brand Memory», Journal of Advertising, 36(4), 75-90.

Lehu, J. M. (2007). "Advergaming: analyse comparative exploratoire de l'attitude des joueurs occasionnels et des har- 
dcore gamers à l'égard du placement de marques dans le jeu vidéo", comunicación presentada en el $6^{\circ}$ Congreso Internacional Paris Venise de Tendencias de Marketing Trends, 26-27 de Enero, París.

Lewis, B. y Porter, L. (2010). «In-game advertising effects: examining player perceptions of advertising schema congruity in a massively multiplayer online role-playing game», Journal of Interactive Advertising, 10(2), 46-60.

Mallinckrodt, V. y Mizerski, D. (2007). "The Effects of Playing an Advergame on Young Children's Perceptions, Preferences, and Requests", Journal of Advertising, 36(2), 87-100.

Martí, J. (2010). Marketing y videojuegos: product placement, in-game advertising y advergaming, Madrid: ESIC.

Molesworth, M. (2006). "Real Brands in Imaginary Worlds: Investigating Players Experiences of Brand Placement in Digital Games, Journal of Consumer Behaviour, 5(4), 355-366.

Moore, E. S. (2006). "It's Child Play: Advergaming and the Online Marketing of Food to Children", The Henry J. Kaiser Family Foundation, <http://www.kff.org/entmedia/upload/ It-s-Child-s-Play-Advergaming-and-the-Online-Marketingof-Food-to-Children-Bios.pdf> (fecha de consulta: 02/02/07) Moran, G. (2004). "Get in the Game," Entrepreneur, (Marzo), 24 .

Nelson, M. R. (2002). "Recall of Brand Placements in Computer/Video Games", Journal of Advertising Research, 42(2), 80-92.

Nelson, M. R., Keum, H. y Yaros, R. A. (2004). “Advertainment or Adcreep? Game Players' Attitudes toward Advertising and Product Placements in Computer Games," Journal of Interactive Advertising, 5:1, <http://www.jiad.org/vol5/ nol/nelson/> (fecha de consulta: 18/06/06)

Nelson, M. R., Yaros, R. A. y Keum, H. (2006). «Examining The Influence Of Telepresence On Spectator And Player Processing Of Real and Fictitious Brands in a Computer Game», Journal of Advertising; 35(4), 87-99.

Nicovich, E. G. (2005). "The Effect of Involvement on Ad Judgment in a Video Game Environment: The Mediating Role of Presence", Journal of Interactive Advertising, 6:1, $<$ http://www.jiad.org/vol6/nol/nicovich/index.htm> (fecha de consulta: 18/06/06)

Paisley, W. J. (1969). "Studying Style as Deviation of Encoding Norm", en The Analysis of Communications Content:
Developments in Scientific Theories and Computer Techniques, Gerbner, G. et alt. (eds.), Nueva York: Willey, 133-146.

Paivio, A. (1979). Imagery and Verbal Processes, Hillsdale, NJ: Lawrence Erlbaum.

Panda, T. K. (2004). "Effectiveness of Product Placements in Indian Films and its effects on Brand Memory and Attitude with Special Reference to Hindi Films", The ICFAI Journal of Marketing Management, (Agosto), 42-56

PQ Media (2008). "Alternative Media Forecast: 20082012", <http://www.pqmedia.com/about-press-20080326amf2008.html> (fecha de consulta: 10/07/08)

PQ Media (2010). "Global Branded Entertainment Marketing Forecast 2010-2014", <http://www.pqmedia.com/ brandedentertainmentforecast2010.html> (fecha de consulta: 05/08/10)

Russell, C. A. (1999). Popular Culture and Persuasión: An Investigation on Product Placements' Effectiveness, Tesis Doctoral, Univesidad de Arizona.

Schneider, L. P. y Cornwell, B. (2005). "Cashing in on crashes via brand placement in computer games: The effects of experience and flow on memory", International Journal of Advertising, 24(3), 321-343.

Vedrashko, I. (2006). Advertising in Computer Games, Tesis de Máster, MIT, <htpp//www.gamesbrandsplay.com> (fecha de consulta: 18/06/07)

Winkler, T. y Buckner, K. (2006). "Receptiveness of Gamers to Embedded Brand Messages in Advergames: Attitudes towards Product Placement", Journal of Interactive Advertising, 7(1), <http://www.jiad.org/vol7/nol/winkler/index. htm> (fecha de consulta: 23/07/07)

Wise, K., Bolls, P. D., Kim, H., Venkataraman A. y Meyer, R. (2008). "Enjoyment of Advergames and Brand Attitudes: The Impact of Thematic Relevante", Journal of Interactive Advertising, 9(1), <http http://www.jiad.org/article107> (consulta: 24/11/2008)

Yang, M., Roskos-Ewoldsen, D. R., Dinu, L. y Arpan, L. M. (2006). "The Effectiveness of 'in-game' advertising: Comparing Collage Students' Explicit and Implicit Memory for Brand Names", Journal of Advertising, 35(4), 143-152.

Youn, S., Lee, M. y Doyle, K. O. (2003). "Lifestyles of Online Gamers: A Psychographic Approach," Journal of Interactive Advertising, 3(2), <http://jiad.org/vol3/no2/youn> (fecha de consulta: 23/07/07) 
